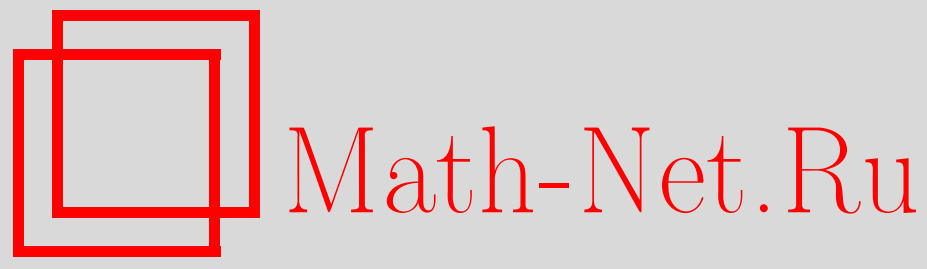

А. К. Алешкявичене, С. В. Нагаев, Переходные явления в случайном блуждании, Теория вероятн. и ее примен., 2003, том 48, выпуск 1, 3-21

DOI: https://doi.org/10.4213/tvp298

Использование Общероссийского математического портала MathNet.Ru подразумевает, что вы прочитали и согласны с пользовательским соглашением

http://www . mathnet.ru/rus/agreement

Параметры загрузки:

IP : 3.80 .181 .102

26 апреля 2023 г., 13:53:57

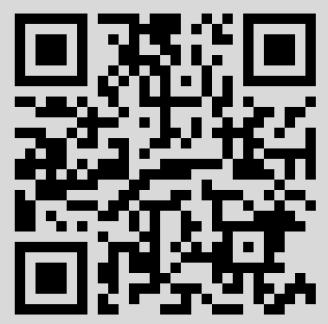




\section{ПЕРЕХОДНЫЕ ЯВЛЕНИЯ В СЛУЧАЙНОМ БЛУЖДАНИИ ${ }^{1)}$}

В статье изучаются предельные распределения максимума сумм $\max _{1 \leqslant k \leqslant n} \sum_{l=1}^{k} \xi_{n, l}$ в схеме серий $\xi_{n, k}, k=1, \ldots, n, n=1,2, \ldots$, независимых одинаково распределенных в отдельной серии случайньгх величин в случаях, когда $a_{n}=\mathbf{E} \xi_{n, k} \rightarrow 0$ и или 1) $a_{n} \sqrt{n} \rightarrow \infty$, или 2) $a_{n} \sqrt{n} \rightarrow-\infty$, или 3) $a_{n} \sqrt{n} \rightarrow 0$ при $n \rightarrow \infty$. Дано прямое доказательство того, что аналитические выражения для предельных законов, полученные ранее разными авторами, совпадают. Кроме того, в этих переходных случаях методом характеристических функций доказана сходимость последовательности распределений максимумов к предельным законам.

Ключевые слова и фразы: схема серий, максимум последовательных сумм, предельные распределения, метод характеристических функций.

Пусть $\xi_{n, k}, k=1, \ldots, n, n=1,2, \ldots$, - последовательность серий независимых случайных величин (сл.в.). Сл.в. $\xi_{n, k}$ одинаково распределены при фиксированном $n$ по закону $F_{n}(x)=\mathbf{P}\left\{\xi_{n, 1}<x\right\}$. Положим

$$
\begin{gathered}
S_{n, k}=\sum_{j=1}^{k} \xi_{n, j}, \quad \bar{S}_{n, k}=\max _{1 \leqslant l \leqslant k} S_{n, l}, \quad k=1, \ldots, n, \\
F_{n, k}(x)=\mathbf{P}\left\{S_{n, k}<x\right\}, \quad \bar{F}_{n, k}(x)=\mathbf{P}\left\{\bar{S}_{n, k}<x\right\} \\
a_{n}=\mathbf{E} \xi_{n, k}, \quad k=1, \ldots, n, \quad \sigma_{n}^{2}=\mathbf{D} \xi_{n, k}, \quad k=1, \ldots, n .
\end{gathered}
$$

Эрдеш и Кац [1] (случай $a_{n}=0$ ) и Вальд [2] (см. также [3]) при исследовании последовательности независимых сл.в. $\xi_{1}, \ldots, \xi_{n}$ с $\mathbf{E} \xi_{k}=a_{k}$ и $\mathbf{D} \xi_{k}=1, k=1, \ldots, n$, показали, что

1) если $a_{n} n^{1 / 2} \rightarrow 0$ при $n \rightarrow \infty$, то

$$
\lim _{n \rightarrow \infty} \mathbf{P}\left\{\bar{S}_{n, n}<x n^{1 / 2}\right\}=\sqrt{\frac{2}{\pi}} \int_{0}^{x} e^{-u^{2} / 2} \mathrm{~d} u ;
$$

${ }^{*}$ Институт математики и информатики, ул. Академийос, 4, 2600 Вильнюс, Литва; e-mail: stat@ktl.mii.lt

${ }^{* *}$ Институт математики им. С. Л. Соболева СО РАН, просп. Академика Коптюга, 4 , 630090 Новосибирск, Россия; e-mail: nagaev@math.nsc.ru

1) Второй автор проводил исследования во время пребывания в Институте математики и информатики в Вильнюсе. 
2) если $a_{n} n^{1 / 2} \rightarrow d$ при $n \rightarrow \infty$ (здесь $d-$ некоторая положительная константа), то при $x \geqslant 0$

$$
\lim _{n \rightarrow \infty} \mathbf{P}\left\{\bar{S}_{n, n}<x n^{1 / 2}\right\}=\int_{d^{2} / 2}^{\infty} \frac{x d e^{x d}}{2 \sqrt{\pi} u^{3 / 2}} e^{-x^{2} d^{2} /(4 u)-u} \mathrm{~d} u
$$

3) если же $a_{n} n^{1 / 2} \rightarrow \infty$ при $n \rightarrow \infty$, то

$$
\lim _{n \rightarrow \infty} \mathbf{P}\left\{\bar{S}_{n, n}<a_{n} n+x n^{1 / 2}\right\}=\frac{1}{\sqrt{2 \pi}} \int_{-\infty}^{x} e^{-u^{2} / 2} \mathrm{~d} u
$$

Более общий результат был получен Ю. В. Прохоровым в работе [5]. Представим этот результат в виде теоремы, формулировка которой заимствована из монографии А. А. Боровкова ([6, гл. 4, § 24, теорема 18]).

Обозначим через $\{\xi(t), t \geqslant 0\}$ стандартный винеровский процесс: $\mathbf{E} \xi(t)=0, \mathbf{E} \xi^{2}(t)=t$.

Теорема ([5], [6]). Пусть выполнены условия

$$
a_{n} \rightarrow 0, \quad \sigma_{n}^{2} \rightarrow \sigma^{2}>0 \quad \text { npu } n \rightarrow \infty
$$

u пусть при любом $\varepsilon>0$

$$
\lim _{n \rightarrow \infty} \int_{|x|>\varepsilon \sqrt{n}} x^{2} \mathrm{~d} F_{n}(x)=0
$$

Тогда если $a_{n} n^{1 / 2} \rightarrow$ a прu $n \rightarrow \infty, a \neq 0 u t=a^{2}$, mo

$$
\begin{aligned}
\lim _{n \rightarrow \infty} \mathbf{P} & \left\{\bar{S}_{n}<\frac{x}{\left|a_{n}\right|}\right\}=\mathbf{P}\left\{\xi(u)<\frac{x-u \operatorname{sign} a}{\sigma}, 0 \leqslant u \leqslant t\right\} \\
= & \left\{\begin{array}{c}
\frac{e^{x / \sigma^{2}}}{\sqrt{2 \pi}} \int_{t \sigma^{2} / x^{2}}^{\infty} u^{-3 / 2} \exp \left\{-\frac{1}{2}\left[\frac{1}{u}+\frac{u x^{2}}{\sigma^{4}}\right]\right\} \mathrm{d} u, \quad a>0, \\
1-e^{-2 x / \sigma^{2}}\left[1-\frac{e^{x / \sigma^{2}}}{\sqrt{2 \pi}}\right] \\
\times \int_{t \sigma^{2} / x^{2}}^{\infty} u^{-3 / 2} \exp \left\{-\frac{1}{2}\left[\frac{1}{\sigma^{2} u}+\frac{u x^{2}}{\sigma^{2}}\right]\right\} \mathrm{d} u, \quad a<0 .
\end{array}\right.
\end{aligned}
$$

Ecлu $a_{n} n^{1 / 2} \rightarrow-\infty$, mo

$$
\lim _{n \rightarrow \infty} \mathbf{P}\left\{\bar{S}_{n}<\frac{x}{\left|a_{n}\right|}\right\}=1-e^{-2 x / \sigma^{2}}
$$

$B$ случаях 1), когда $\left.a_{n} n^{1 / 2} \rightarrow \infty n p u n \rightarrow \infty, u 2\right), \kappa о г д а ~ n a_{n}^{2} \rightarrow 0$ при $n \rightarrow \infty$, имеют место соотношения (3) и (1) соответственно. 
3 а м е ч а н и е 1. Утверждения (2) и (7) эквивалентны. Действительно, из соотношений (6)-(8), поменяв нормировку и положив $d_{n}=\left|a_{n}\right| \sqrt{n} / \sigma_{n}, d=\lim _{n \rightarrow \infty} d_{n}=|a| / \sigma$, получаем

$$
\begin{gathered}
\lim _{n \rightarrow \infty} \mathbf{P}\left\{\frac{\bar{S}_{n, n}}{\sigma_{n} \sqrt{n}}<x\right\}=\mathbf{P}\{\xi(u)+u d \operatorname{sign} a<x, 0 \leqslant u \leqslant 1\} \\
=\left\{\begin{array}{l}
\frac{1}{\sqrt{2 \pi}} \int_{1 / x^{2}}^{\infty} u^{-3 / 2} \exp \left\{-\frac{1}{2}\left(\frac{1}{u}+u x^{2} d^{2}\right)\right\} \mathrm{d} u, \quad a>0 \\
1-e^{-2 x d}\left[1-\frac{e^{x d}}{\sqrt{2 \pi}}\right] \\
\times \int_{1 / x^{2}}^{\infty} u^{-3 / 2} \exp \left\{-\frac{1}{2}\left(\frac{1}{u}+u x^{2} d^{2}\right)\right\} \mathrm{d} u, \quad a<0 .
\end{array}\right.
\end{gathered}
$$

Отсюда, сделав замену переменной $v=u x^{2} d^{2} / 2$, находим

$$
\lim _{n \rightarrow \infty} \mathbf{P}\left\{\frac{\bar{S}_{n, n}}{\sigma_{n} \sqrt{n}}<x\right\}= \begin{cases}\tilde{V}_{1}(x), & a>0 \\ \tilde{V}_{2}(x), & a<0\end{cases}
$$

где

$$
\begin{aligned}
& \widetilde{V}_{1}(x)=\frac{1}{2 \sqrt{\pi}} x d e^{x d} \int_{d^{2} / 2}^{\infty} u^{-3 / 2} e^{-x^{2} d^{2} /(4 u)-u} \mathrm{~d} u, \quad x \geqslant 0, \\
& \tilde{V}_{2}(x)=1-e^{-2 x d}\left[1-\frac{1}{2 \sqrt{\pi}} x d e^{x d}\right] \int_{d^{2} / 2}^{\infty} u^{-3 / 2} e^{-x^{2} d^{2} /(4 u)-u} \mathrm{~d} u, \quad x \geqslant 0,
\end{aligned}
$$

и $\widetilde{V}_{1}(x ; d)=\widetilde{V}_{2}(x ; d)=0, x<0$. Замечаем, что соотношение $(11)$ при $\sigma_{n}=1$ совпадает с соотношением (2). Следовательно, утверждения (2) и (7) эквивалентны.

Положим

$$
\Phi(x)=\frac{1}{\sqrt{2 \pi}} \int_{-\infty}^{x} e^{-u^{2} / 2} \mathrm{~d} u, \quad \Psi(x)=1-\Phi(x) .
$$

Пусть $\bar{a}$ и $b>0$ - постоянные. Тогда из (10) и из соотношения (см. [4])

$$
\begin{aligned}
& \mathbf{P}\left\{\sup _{0 \leqslant u \leqslant T}(\xi(u)-\bar{a} u) \geqslant b\right\} \\
& \quad=\Psi\left((\bar{a} T+b) T^{-1 / 2}\right)+e^{-2 \bar{a} b} \Phi\left((\bar{a} T-b) T^{-1 / 2}\right)
\end{aligned}
$$

вытекает (см. также [8]), что

$$
\lim _{n \rightarrow \infty} \mathbf{P}\left\{\frac{\bar{S}_{n}}{\sigma_{n} \sqrt{n}}<x\right\}= \begin{cases}V_{1}(x ; d), & a_{n}>0 \\ V_{2}(x ; d), & a_{n}<0\end{cases}
$$


где

$$
\begin{aligned}
& V_{1}(x ; d)=\frac{1}{\sqrt{2 \pi}} \int_{-\infty}^{x-d} e^{-u^{2} / 2} \mathrm{~d} u-\frac{1}{\sqrt{2 \pi}} e^{2 x d} \int_{-\infty}^{-x-d} e^{-u^{2} / 2} \mathrm{~d} u \\
& V_{2}(x ; d)=\frac{1}{\sqrt{2 \pi}} \int_{-\infty}^{x+d} e^{-u^{2} / 2} \mathrm{~d} u-\frac{1}{\sqrt{2 \pi}} e^{-2 x d} \int_{-\infty}^{-x+d} e^{-u^{2} / 2} \mathrm{~d} u .
\end{aligned}
$$

Тем же самым способом, опираясь на соотношения (10) и (13), можно убедиться в справедливости соотношений (1), (3) и (9).

Отметим, что соотношения (7), (8), (14) и (15) были ранее доказаны прямыми вероятностными методами. Ниже мы приведем доказательство соотношений (14) и (15) методом характеристических функций (см. ниже теорему 1).

3 а м е ч а н и е 2. Сравнивая соотношения (11) и (14), а также (12) и (15), получаем функциональные тождества:

$$
\begin{aligned}
& \frac{1}{2 \sqrt{\pi}} x d e^{x d} \int_{d^{2} / 2}^{\infty} u^{-3 / 2} e^{-x^{2} d^{2} /(4 u)-u} \mathrm{~d} u \\
& \quad=\frac{1}{\sqrt{2 \pi}} \int_{-\infty}^{x-d} e^{-u^{2} / 2} \mathrm{~d} u-\frac{1}{\sqrt{2 \pi}} e^{2 x d} \int_{-\infty}^{-x-d} e^{-u^{2} / 2} \mathrm{~d} u, \quad x>0 \\
& 1-e^{-2 x d}\left[1-\frac{1}{2 \sqrt{\pi}} x d e^{x d} \int_{d^{2} / 2}^{\infty} u^{\left.-3 / e^{-x^{2} d^{2} /(4 u)-u} \mathrm{~d} u\right]}\right. \\
& =\frac{1}{\sqrt{2 \pi}} \int_{-\infty}^{x+d} e^{-u^{2} / 2} \mathrm{~d} u-\frac{1}{\sqrt{2 \pi}} e^{-2 x d} \int_{-\infty}^{-x+d} e^{-u^{2} / 2} \mathrm{~d} u, \quad x>0
\end{aligned}
$$

Но эти тождества означают, что функция распределения $V_{1}(x ; d)$ совпадает с функцией распределения $\widetilde{V}_{1}(x ; d)$, а $V_{2}(x ; d)$ совпадает с $\widetilde{V}_{2}(x ; d)$.

Естественно возникает задача: вывести тождества (16) и (17) непосредственно, не опираясь на предельные соотношения (11), (14) и $(12),(15)$.

Мы решаем эту задачу с помощью метода характеристических функций. Конечно, хотелось бы дать более элементарное доказательство, включающее лишь интегрирование и дифференцирование, но это сделать авторам не удалось.

Д о к а 3 а т е л ь с т в о т о ж д е с т в а (16). Мы не будем вычислять характеристическую функцию распределения $\tilde{V}_{1}(x ; d)$. Вместо этого мы найдем преобразование Фурье-Стилтьеса функции

$$
\bar{V}_{1}(x ; d)=\frac{x d e^{x d}}{2 \sqrt{\pi}} \int_{d^{2} / 2}^{\infty} u^{-3 / 2} e^{-x^{2} d^{2} /(4 u)-u} \mathrm{~d} u,
$$

определенной для всех $x \in \mathbf{R}_{1}$ и совпадающей с $\tilde{V}_{1}(x ; d)$ при $x \geqslant 0$. Затем, при переходе обратно к прообразу, возьмем только часть прообраза, 
определенную для $x \geqslant 0$, поскольку только эта часть будет соответствовать интересующему нас распределению $\widetilde{V}_{1}(x ; d), x \geqslant 0$. Имеем

$$
\begin{aligned}
& \int_{-\infty}^{\infty} e^{i t x} d \bar{V}_{1}(x ; d) \\
& =\frac{d}{2 \sqrt{\pi}} \int_{d^{2} / 2}^{\infty} \frac{e^{-u}}{u^{3 / 2}} \mathrm{~d} u \int_{-\infty}^{\infty} e^{i t x}\left[1+x\left(d-\frac{d^{2}}{2 u} x\right)\right] e^{x d-x^{2} d^{2} /(4 u)} \mathrm{d} x \\
& =\frac{d}{2 \sqrt{\pi}} \int_{d^{2} / 2}^{\infty} u^{-3 / 2} \mathrm{~d} u \int_{-\infty}^{\infty} e^{i t x}\left[1+x d-\frac{x^{2} d^{2}}{2 u}\right] e^{-1 / 2} \frac{(x-2 u / d)^{2}}{2 u / d^{2}} \mathrm{~d} x \\
& =\frac{d}{2 \sqrt{\pi}} \int_{d^{2} / 2}^{\infty} \frac{\mathrm{d} u}{u^{3 / 2}}\left[\exp \left\{i t \frac{2 u}{d}-\frac{2 u}{d^{2}} \frac{t^{2}}{2}\right\}\right. \\
& +\frac{d}{i} \frac{\mathrm{d}}{\mathrm{d} t}\left(\exp \left\{i t \frac{2 u}{d}-\frac{2 u}{d^{2}} \frac{t^{2}}{2}\right\}\right) \\
& \left.+\frac{d^{2}}{2 u} \frac{\mathrm{d}^{2}}{\mathrm{~d} t^{2}}\left(\exp \left\{i t \frac{2 u}{d}-\frac{2 u}{d^{2}} \frac{t^{2}}{2}\right\}\right)\right] \frac{2 \sqrt{\pi u}}{d} \\
& =\int_{d^{2} / 2}^{\infty} \frac{1}{u}\left[1+\frac{d}{i}\left(i \frac{2 u}{d}-\frac{2 u}{d^{2}} t\right)+\frac{d^{2}}{2 u}\left(-\frac{2 u}{d^{2}}+\left(i \frac{2 u}{d}-\frac{2 u}{d^{2}} t\right)^{2}\right)\right] \\
& \times \exp \left\{i t \frac{2 u}{d}-\frac{2 u}{d^{2}} \frac{t^{2}}{2}\right\} \mathrm{d} u \\
& =\left(\frac{2 t^{2}}{d^{2}}-\frac{2 i t}{d}\right) \int_{d^{2} / 2}^{\infty} \exp \left\{i t \frac{2 u}{d}-\frac{2 u}{d^{2}} \frac{t^{2}}{2}\right\} \mathrm{d} u \\
& =\left(-\frac{t^{2}}{i t d-t^{2} / 2}+\frac{2 d}{2 d+i t}\right) e^{-t^{2} / 2+i t d}=\frac{2 i t+2 d}{2 d+i t} e^{-t^{2} / 2+i t d} \\
& =\left(2-\frac{2 d}{2 d+i t}\right) e^{-t^{2} / 2+i t d}
\end{aligned}
$$

Заметим, что $2 d /(i t+2 d)$ является характеристической функцией плотности

$$
q(u)= \begin{cases}0, & u \geqslant 0, \\ 2 d e^{2 u d}, & u<0 .\end{cases}
$$

Но тогда функция $2 d(i t+2 d)^{-1} \exp \left\{-t^{2} / 2+i t d\right\}$ является характеристической функцией свертки

$$
\frac{2 d}{\sqrt{2 \pi}} \int_{-\infty}^{0} e^{-(x-d-u)^{2} / 2} e^{2 u d} \mathrm{~d} u
$$

двух плотностей $q(x)$ и $(1 / \sqrt{2 \pi}) e^{-(x-d)^{2} / 2}$, которая после замены переменной $u-x=v+d$ преобразуется в функцию $(2 d / \sqrt{2 \pi}) e^{2 x d} \int_{-\infty}^{-x-d} e^{-v^{2} / 2} \mathrm{~d} v$. Тогда плотностью (положительной частью) прообраза «характеристической функции» (18), соответствующей функции распределения $\tilde{V}_{1}(x ; d), x \geqslant 0$, будет функция

$$
\tilde{p}_{1}(x)=\frac{2}{\sqrt{2 \pi}} e^{-(x-d)^{2} / 2}-\frac{2 d}{\sqrt{2 \pi}} e^{2 x d} \int_{-\infty}^{-x-d} e^{-u^{2} / 2} \mathrm{~d} u, \quad x \geqslant 0 .
$$


Но эта функция совпадает с плотностью закона $V_{1}(x ; d)$, т.е. с функцией

$$
\begin{aligned}
p_{1}(x) & =\frac{\mathrm{d}}{\mathrm{d} x} V_{1}(x ; d)=\frac{\mathrm{d}}{\mathrm{d} x}\left(\frac{1}{\sqrt{2 \pi}} \int_{-\infty}^{x-d} e^{-u^{2} / 2} \mathrm{~d} u-\frac{e^{2 x d}}{\sqrt{2 \pi}} \int_{-\infty}^{-x-d} e^{-u^{2} / 2} \mathrm{~d} u\right) \\
& =\frac{2}{\sqrt{2 \pi}} e^{-(x-d)^{2} / 2}-\frac{2 d}{\sqrt{2 \pi}} e^{2 x d} \int_{-\infty}^{-x-d} e^{-u^{2} / 2} \mathrm{~d} u, \quad x \geqslant 0 .
\end{aligned}
$$

Следовательно, законы $\widetilde{V}_{1}(x ; d)$ и $V_{1}(x ; d)$ совпадают и тождество (16) доказано.

Доказ а т ель с т в о т ож д е с т в а (17). Пусть $\widetilde{p}_{2}(x ; d)$ обозначает плотность закона $\widetilde{V}_{2}(x ; d)$. Тогда

$$
\begin{aligned}
\widetilde{p}_{2}(x ; d)= & \frac{\mathrm{d}}{\mathrm{d} x} \widetilde{V}_{2}(x ; d)=2 d e^{-2 x d} \\
& +\frac{\mathrm{d}}{\mathrm{d} x}\left(\frac{x d e^{-x d}}{2 \sqrt{\pi}} \int_{d^{2} / 2}^{\infty} u^{-3 / 2} e^{-\left(x^{2} d^{2} /(4 u)+u\right)} \mathrm{d} u\right), \quad x \geqslant 0 .
\end{aligned}
$$

Для преобразования второго слагаемого правой части соотношения (19), которое в дальнейшем будет обозначаться через $\widetilde{p}_{2,2}(x ; d)$, будем пользоваться теми рассуждениями, которые проводились при вычислении преобразования Фурье-Стилтьеса закона $\widetilde{V}_{1}(x ; d)$.

Итак, сначала найдем преобразование Фурье функции

$$
\bar{p}_{2,2}(x ; d)=\frac{\mathrm{d}}{\mathrm{d} x}\left(\frac{x d e^{-x d}}{2 \sqrt{\pi}} \int_{d^{2} / 2}^{\infty} u^{-3 / 2} e^{-x^{2} d^{2} /(4 u)-u} \mathrm{~d} u\right),
$$

определенной для всех $x \in(-\infty, \infty)$ и совпадающей с $\widetilde{p}_{2,2}(x ; d)$ при $x \geqslant 0$. Затем, при переходе обратно к прообразу, возьмем только часть прообраза, определенную для $x \geqslant 0$. Аналогично (18) получаем

$$
\begin{aligned}
& \int_{-\infty}^{\infty} e^{i t x} \bar{p}_{2,2}(x ; d) \mathrm{d} x \\
& =\frac{d}{2 \sqrt{\pi}} \int_{d^{2} / 2}^{\infty} \frac{e^{-u}}{u^{3 / 2}} \mathrm{~d} u \int_{-\infty}^{\infty} e^{i t x}\left(1-x d-\frac{d^{2}}{2 u} x^{2}\right) \\
& \quad \times \exp \left\{-\frac{1}{2} \frac{(x+2 u / d)^{2}}{2 u / d^{2}}\right\} \mathrm{d} x \\
& =\int_{d^{2} / 2}^{\infty} \frac{1}{u}\left[\exp \left\{-\frac{2 u}{d} i t-\frac{2 u}{d^{2}} \frac{t^{2}}{2}\right\}-\frac{d}{i} \frac{\mathrm{d}}{\mathrm{d} t} \exp \left\{-\frac{2 u}{d} i t-\frac{2 u}{d^{2}} \frac{t^{2}}{2}\right\}\right. \\
& \left.\quad+\frac{d^{2}}{2 u} \frac{\mathrm{d}^{2}}{\mathrm{~d} t^{2}} \exp \left\{-\frac{2 u}{d} i t-\frac{2 u}{d^{2}} \frac{t^{2}}{2}\right\}\right] \mathrm{d} u \\
& =\int_{d^{2} / 2}^{\infty}\left(\frac{2}{d} i t+\frac{2}{d^{2}} t^{2}\right) \exp \left\{-i t \frac{2 u}{d}-\frac{2 u}{d^{2}} \frac{t^{2}}{2}\right\} \mathrm{d} u \\
& =\left(2-\frac{2 d}{2 d-i t}\right) \exp \left\{-i t d-\frac{t^{2}}{2}\right\} .
\end{aligned}
$$


Отметим, что функция $2 d /(2 d-i t)$ является характеристической функцией плотности

$$
q_{2}(x)= \begin{cases}2 d e^{-2 d x}, & x \geqslant 0 \\ 0, & x<0 .\end{cases}
$$

Но тогда функция $2 d(2 d-i t)^{-1} \exp \left\{-i t d-t^{2} / 2\right\}$ является преобразованием Фурье свертки

$$
\frac{2 d}{\sqrt{2 \pi}} \int_{0}^{\infty} e^{-(x+d-u)^{2} / 2} e^{-2 u d} \mathrm{~d} u
$$

двух плотностей $q_{2}(x)$ и $(1 / \sqrt{2 \pi}) e^{-(x+d)^{2} / 2}$. Эта свертка после замены переменной $u=v-d+x$ преобразуется в функцию

$$
\frac{2 d}{\sqrt{2 \pi}} e^{-2 d x} \int_{-x+d}^{\infty} e^{-v^{2} / 2} \mathrm{~d} v, \quad-\infty<x<\infty
$$

Отсюда и из соотношений (19), (20) вытекает, что плотностью закона $\widetilde{V}_{2}(x ; d)$ является функция

$$
\begin{aligned}
\widetilde{p}_{2}(x ; d) & =2 d e^{-2 x d}+\sqrt{\frac{2}{\pi}} e^{-(x+d)^{2} / 2}-\frac{2 d}{\sqrt{2 \pi}} e^{-2 d x} \int_{-x+d}^{\infty} e^{-v^{2} / 2} \mathrm{~d} v \\
& =\sqrt{\frac{2}{\pi}} e^{-(x+d)^{2} / 2}+2 d e^{-2 d x} \frac{1}{\sqrt{2 \pi}} \int_{-\infty}^{-x+d} e^{-v^{2} / 2} \mathrm{~d} v, \quad x \geqslant 0 .
\end{aligned}
$$

С другой стороны, нетрудно убедиться, что эта функция совпадает с плотностью закона $V_{2}(x ; d)$, т.е. с функцией

$$
\begin{aligned}
p_{2}(x)= & \frac{\mathrm{d}}{\mathrm{d} x} V_{2}(x ; d) \\
= & \frac{\mathrm{d}}{\mathrm{d} x}\left(\frac{1}{\sqrt{2 \pi}} \int_{-\infty}^{x+d} e^{-u^{2} / 2} \mathrm{~d} u-e^{-2 x d} \frac{1}{\sqrt{2 \pi}} \int_{-\infty}^{-x+d} e^{-u^{2} / 2} \mathrm{~d} u\right) \\
= & \frac{1}{\sqrt{2 \pi}} e^{-(x+d)^{2} / 2}+2 d e^{-2 x d} \frac{1}{\sqrt{2 \pi}} \int_{-\infty}^{-x+d} e^{-u^{2} / 2} \mathrm{~d} u \\
& +e^{-2 x d} \frac{1}{\sqrt{2 \pi}} e^{-(x-d)^{2} / 2} \\
= & \sqrt{\frac{2}{\pi}} e^{-(x+d)^{2} / 2}+\frac{2 d}{\sqrt{2 \pi}} e^{-2 x d} \int_{-\infty}^{-x+d} e^{-u^{2} / 2} \mathrm{~d} u, \quad x \geqslant 0 .
\end{aligned}
$$

Таким образом, законы $V_{2}(x ; d)$ и $\widetilde{V}_{2}(x ; d)$ совпадают и, следовательно, тождество (17) доказано.

Д о к а за т ель с т в о с о о т н ош ен и й (14) и (15). Выше мы упомянули, что докажем соотношения (14) и (15) методом характеристических функций. Мы сформулируем наши результаты в виде следуюшей теоремы. 
Теорема 1. Пусть выполнены условия (4) и (5). Тогда если $a_{n} n^{1 / 2} \rightarrow$ a nри $n \rightarrow \infty, d_{n}:=\left|a_{n}\right| n^{1 / 2} / \sigma_{n} u d_{n} \rightarrow d$, то равномерно по всем $d \in\left[\varepsilon_{1}, A\right]$ имеют место соотношения (14) и (15) (здесь $\varepsilon_{1}$ и Aсколь угодно малое и сколь угодно большое положительные числа).

Прежде чем приступить к доказательству теоремы 1 , мы приведем несколько лемм.

Лемма 1. Для характеристической функиии $f_{V_{1}}(t)$ распределения

$$
V_{1}(x ; d)=\frac{1}{\sqrt{2 \pi}} \int_{-\infty}^{x-d} e^{-u^{2} / 2} \mathrm{~d} u-\frac{1}{\sqrt{2 \pi}} e^{2 x d} \int_{-\infty}^{-x-d} e^{-u^{2} / 2} \mathrm{~d} u
$$

имеет место пред̀ставление

$$
\begin{aligned}
f_{V_{1}}(t)= & \left(1-\frac{d}{i t+2 d}\right) e^{i t d-t^{2} / 2}+\frac{d}{i t+2 d}\left(1-\sqrt{\frac{2}{\pi}} \int_{0}^{d} e^{-u^{2} / 2} \mathrm{~d} u\right) \\
& +\frac{1}{\sqrt{2 \pi}}\left(i t+\frac{d^{2}}{i t+2 d}\right) e^{-d^{2} / 2} \int_{0}^{1} e^{(d+i t)^{2} u / 2} \frac{\mathrm{d} u}{\sqrt{1-u}} .
\end{aligned}
$$

Док азатель с тв о. Имеем

$$
\begin{aligned}
f_{V_{1}}(t)= & \int_{0}^{\infty} e^{i t x} \mathrm{~d} V_{1}(x ; d) \\
= & \frac{1}{\sqrt{2 \pi}} \int_{0}^{\infty} e^{i t x} e^{-(x-d)^{2} / 2} \mathrm{~d} x \\
& -\frac{1}{\sqrt{2 \pi}} \int_{0}^{\infty} e^{i t x} \mathrm{~d}_{x}\left(e^{2 x d} \int_{-\infty}^{-x} e^{-(u-d)^{2} / 2} \mathrm{~d} u\right) \\
= & \sqrt{\frac{2}{\pi}} \int_{0}^{\infty} e^{i t x} e^{-(x-d)^{2} / 2} \mathrm{~d} x \\
& -\frac{2 d}{\sqrt{2 \pi}} \int_{0}^{\infty} e^{i t x} e^{2 d x}\left(\int_{-\infty}^{-x} e^{-(u-d)^{2} / 2} \mathrm{~d} u\right) \mathrm{d} x \\
= & \sqrt{\frac{2}{\pi}}\left(1-\frac{d}{i t+2 d}\right) \int_{0}^{\infty} e^{i t x} e^{-(x-d)^{2} / 2} \mathrm{~d} x \\
& +\sqrt{\frac{2}{\pi}} \frac{d}{i t+2 d} \int_{-\infty}^{0} e^{-(u-d)^{2} / 2} \mathrm{~d} u .
\end{aligned}
$$

Для преобразования первого интеграла в правой части (22) воспользуемся следующей формулой (см. [10, с. 321, формула $3.322(2)]$ ):

$$
\begin{aligned}
& \int_{0}^{\infty} \exp \left\{-\frac{x^{2}}{4 \beta}-\gamma x\right\} \mathrm{d} x=\sqrt{\pi \beta} \exp \left\{\beta \gamma^{2}\right\}[1-\tilde{\Phi}(\gamma \sqrt{\beta})], \\
& \operatorname{Re} \beta>0, \quad \widetilde{\Phi}(x)=\frac{2}{\sqrt{\pi}} \int_{0}^{x} e^{-u^{2} / 2} \mathrm{~d} u=\sqrt{\frac{2}{\pi}} \int_{0}^{\sqrt{2} x} e^{-u^{2} / 2} \mathrm{~d} u .
\end{aligned}
$$


Применяя (23) при $\beta=\frac{1}{2}$ и $\gamma=-(d+i t)$ и поочередно сделав три очевидные замены переменных, получаем

$$
\begin{aligned}
I_{1} & =\int_{0}^{\infty} e^{i t x} e^{-(x-d)^{2} / 2} \mathrm{~d} x=\frac{1}{\sqrt{2 \pi}} e^{-d^{2} / 2} \int_{0}^{\infty} e^{-x^{2} / 2+x(d+i t)} \mathrm{d} x \\
& =\frac{1}{2} e^{-d^{2} / 2} e^{(d+i t)^{2} / 2}\left[1-\sqrt{\frac{2}{\pi}} \int_{0}^{-(d+i t)} e^{-u^{2} / 2} \mathrm{~d} u\right] \\
& =\frac{1}{2} e^{i t d-t^{2} / 2}\left[1+\sqrt{\frac{2}{\pi}} \int_{0}^{1} e^{-\left(u^{2} / 2\right)(d+i t)^{2}}(i t+d) \mathrm{d} u\right] \\
& =\frac{1}{2} e^{i t d-t^{2} / 2}\left[1+\sqrt{\frac{2}{\pi}}(i t+d) \int_{0}^{1} e^{-(d+i t)^{2} u / 2} \frac{\mathrm{d} u}{2 \sqrt{u}}\right] \\
& =\frac{1}{2} e^{i t d-t^{2} / 2}\left[1+\frac{1}{\sqrt{2 \pi}}(i t+d) e^{-(d+i t)^{2} / 2} \int_{0}^{1} e^{(d+i t)^{2} u / 2} \frac{\mathrm{d} u}{\sqrt{1-u}}\right] .
\end{aligned}
$$

Второй интеграл в правой части (22) перепишем следующим образом:

$$
\begin{aligned}
I_{2} & =\sqrt{\frac{2}{\pi}} \frac{d}{i t+2 d} \int_{-\infty}^{0} e^{-(u-d)^{2} / 2} \mathrm{~d} u=\sqrt{\frac{2}{\pi}} \frac{d}{i t+2 d} \int_{-\infty}^{-d} e^{-u^{2} / 2} \mathrm{~d} u \\
& =\sqrt{\frac{2}{\pi}} \frac{d}{i t+2 d}\left(\sqrt{\frac{2 \pi}{2}}-\int_{-d}^{0} e^{-u^{2} / 2} \mathrm{~d} u\right) \\
& =\frac{d}{i t+2 d}-\sqrt{\frac{2}{\pi}} \frac{d}{i t+2 d} \int_{0}^{d} e^{-u^{2} / 2} \mathrm{~d} u .
\end{aligned}
$$

Таким образом, из (22), (24) и (25) следует (21). Лемма 1 доказана.

Лемма 2. Для характеристической функции $f_{V_{2}}(t)$ распределения

$$
V_{2}(x ; d)=\frac{1}{\sqrt{2 \pi}} \int_{-\infty}^{x+d} e^{-u^{2} / 2} \mathrm{~d} u-\frac{1}{\sqrt{2 \pi}} e^{-2 x d} \int_{-\infty}^{-x+d} e^{-u^{2} / 2} \mathrm{~d} u
$$

имеет место представление

$$
\begin{aligned}
f_{V_{2}}(t)= & \left(1+\frac{d}{i t-2 d}\right) e^{-i t d-t^{2} / 2}+\frac{1}{\sqrt{2 \pi}}\left(i t+\frac{d^{2}}{i t-2 d}\right) e^{-d^{2} / 2} \\
& \times \int_{0}^{1} e^{(i t-d)^{2} u / 2} \frac{\mathrm{d} u}{\sqrt{1-u}}-\frac{d}{i t-2 d}\left(1+\sqrt{\frac{2}{\pi}} \int_{0}^{d} e^{-u^{2} / 2} \mathrm{~d} u\right) .
\end{aligned}
$$

Д ок аз а тель с тв о. Имеем

$$
\begin{aligned}
f_{V_{2}}(t)= & \int_{0}^{\infty} e^{i t x} \mathrm{~d} V_{2}(x ; d)=\sqrt{\frac{2}{\pi}} \int_{0}^{\infty} e^{i t x} e^{-(x+d)^{2} / 2} \mathrm{~d} x \\
& +\frac{2 d}{\sqrt{2 \pi}} \int_{0}^{\infty} e^{i t x-2 x d}\left(\int_{-\infty}^{-x} e^{-(u+d)^{2} / 2} \mathrm{~d} u\right) \mathrm{d} x
\end{aligned}
$$




$$
\begin{aligned}
=\sqrt{\frac{2}{\pi}}( & +\frac{d}{i t-2 d} \int_{0}^{\infty} e^{i t x} e^{-(x+d)^{2} / 2} \mathrm{~d} x \\
& \left.-\sqrt{\frac{2}{\pi}} \frac{d}{i t-2 d} \int_{-\infty}^{0} e^{-(u+d)^{2} / 2} \mathrm{~d} u\right) .
\end{aligned}
$$

Аналогично преобразованию интеграла $I_{1}$ из (22) (см. (24)) получаем, что

$$
\begin{aligned}
& \frac{1}{\sqrt{2 \pi}} \int_{0}^{\infty} e^{i t x} e^{-(x+d)^{2} / 2} \mathrm{~d} x \\
& =\frac{1}{2} e^{-i t d-t^{2} / 2}\left[1+\frac{1}{\sqrt{2 \pi}}(i t-d) e^{-(i t-d)^{2} / 2} \int_{0}^{1} e^{(i t-d)^{2} / 2} \frac{\mathrm{d} u}{\sqrt{1-u}}\right]
\end{aligned}
$$

Далее, имеем

$$
\begin{aligned}
& -\sqrt{\frac{2}{\pi}} \frac{d}{i t-2 d} \int_{-\infty}^{0} e^{-(u+d)^{2} / 2} \mathrm{~d} u=-\sqrt{\frac{2}{\pi}} \frac{d}{i t-2 d} \int_{-\infty}^{d} e^{-u^{2} / 2} \mathrm{~d} u \\
& \quad=-\sqrt{\frac{2}{\pi}} \frac{d}{i t-2 d}\left[\frac{\sqrt{2 \pi}}{2}+\int_{0}^{d} e^{-u^{2} / 2} \mathrm{~d} u\right] .
\end{aligned}
$$

Подставляя (28) и (29) в (27), убеждаемся в справедливости соотношения (26). Лемма 2 доказана.

Далее, для исследования предельного поведения характеристической функции сл.в. $\bar{S}_{n, n}$ нам нужно будет знать асимптотическое поведение величин

$$
\bar{a}_{n, k}=\int_{-\infty}^{0-} x \mathrm{~d} \bar{F}_{n, k}(x) \quad \text { и } \quad \bar{b}_{n, k}=\int_{-\infty}^{0-} x^{2} \mathrm{~d} \bar{F}_{n, k}(x) .
$$

Лемма 3. Если выполнены условия теоремы 1, то

$$
\bar{a}_{n, k}=-\frac{\sigma_{n}}{\sqrt{2 \pi k}} e^{-a_{n}^{2} k /\left(2 \sigma_{n}^{2}\right)}+a_{n} \Phi\left(-\frac{a_{n} \sqrt{k}}{\sigma_{n}}\right)+\frac{\varepsilon_{n, k}}{\sqrt{k}},
$$

где $\varepsilon_{n, k} \rightarrow 0$ npu $n \rightarrow \infty, k \rightarrow \infty$.

Д о к а з а т е л с т в о. В работе $[8$, формула (1.7)] показано, что

$$
\bar{a}_{n, k}=\frac{1}{k} \int_{-\infty}^{0-} x \mathrm{~d} F_{n, k}(x) \text {. }
$$

Отсюда

$$
\begin{aligned}
\bar{a}_{n, k}= & -\frac{\sigma_{n}}{\sqrt{k}} \int_{-\infty}^{-a_{n} \sqrt{k} / \sigma_{n}} F_{n, k}\left(x \sigma_{n} \sqrt{k}+a_{n} k\right) \mathrm{d} x \\
= & -\frac{\sigma_{n}}{\sqrt{k}} \int_{-\infty}^{-a_{n} \sqrt{k} / \sigma_{n}} \Phi(x) \mathrm{d} x \\
& +\frac{\sigma_{n}}{\sqrt{k}} \int_{-\infty}^{-a_{n} \sqrt{k} / \sigma_{n}}\left[\Phi(x)-F_{n, k}\left(x \sigma_{n} \sqrt{k}+a_{n} k\right)\right] \mathrm{d} x
\end{aligned}
$$


Интегрируя по частям, находим

$$
-\frac{\sigma_{n}}{\sqrt{k}} \int_{-\infty}^{-a_{n} \sqrt{k} / \sigma_{n}} \Phi(x) \mathrm{d} x=-\frac{\sigma_{n}}{\sqrt{2 \pi k}} e^{-a_{n}^{2} k /\left(2 \sigma_{n}^{2}\right)}+a_{n} \Phi\left(-\frac{a_{n} \sqrt{k}}{\sigma_{n}}\right) .
$$

Оценим теперь второе слагаемое в правой части соотношения (31). Для любого $L<-a_{n} \sqrt{k} / \sigma_{n}$ положим

$$
\begin{aligned}
\int_{-\infty}^{-a_{n} \sqrt{k} / \sigma_{n}}\left|\Phi(x)-F_{n, k}\left(x \sigma_{n} \sqrt{k}+a_{n} k\right)\right| \mathrm{d} x & =\int_{-\infty}^{-L}+\int_{-L}^{-a_{n} \sqrt{k} / \sigma_{n}} \\
& =J_{1}+J_{2} .
\end{aligned}
$$

Согласно центральной предельной теореме, которая имеет место в силу условий (4) и (5), для любого фиксированного $L$

$$
\lim _{n \rightarrow \infty, k \rightarrow \infty} J_{2}=0 \text {. }
$$

С другой стороны,

$$
\begin{aligned}
\int_{-\infty}^{-L} \Phi(x) \mathrm{d} x & <\frac{1}{|L|} \int_{-\infty}^{-L}|x| \Phi(x) \mathrm{d} x<\frac{1}{|L|} \int_{-\infty}^{0}|x| \Phi(x) \mathrm{d} x \\
& =\frac{1}{2|L|} \int_{-\infty}^{0} x^{2} \mathrm{~d} \Phi(x)=\frac{1}{4|L|}
\end{aligned}
$$

Аналогично

$$
\int_{-\infty}^{-L} F_{n, k}\left(x \sigma_{n} \sqrt{k}+a_{n} k\right) \mathrm{d} x<\frac{1}{2|L|}
$$

Таким образом,

$$
J_{1}<\frac{3}{4|L|}
$$

Так как $L$ можно выбрать сколь угодно большим по абсолютной величине, то в силу (33)-(35) можем утверждать, что

$$
\frac{\sigma_{n}}{\sqrt{k}} \int_{-\infty}^{-a_{n} \sqrt{k} / \sigma_{n}}\left[\Phi(x)-F_{n, k}\left(x \sigma_{n} \sqrt{k}+a_{n} k\right)\right] \mathrm{d} x=\frac{\sigma_{n} \varepsilon_{n, k}}{\sqrt{k}}
$$

где $\varepsilon_{n, k} \rightarrow 0$ при $n \rightarrow \infty$ и $k \rightarrow \infty$.

Из (31), (32) и (36) следует утверждение леммы 3.

Лемма 4. Если выполнены условия теоремь 1, то $\left|\bar{a}_{n, k}\right|<\sigma_{n} / \sqrt{k}$.

Д ок а з а т л ь с т в о. Согласно (30),

$$
\begin{aligned}
\left|\bar{a}_{n, k}\right| & <\frac{\sigma_{n}}{\sqrt{k}} \int_{-\infty}^{0} F_{n, k}\left(x \sigma_{n} \sqrt{k}+a_{n} k\right) \mathrm{d} x<\frac{\sigma_{n}}{\sqrt{k}} \mathbf{E}\left|\frac{S_{n, k}-a_{n} k}{\sigma_{n} \sqrt{k}}\right| \\
& \leqslant \frac{\sigma_{n}}{\sqrt{k}}\left(\mathbf{E}\left(\frac{S_{n, k}-a_{n} k}{\sigma_{n} \sqrt{k}}\right)^{2}\right)^{1 / 2}=\frac{\sigma_{n}}{\sqrt{k}} .
\end{aligned}
$$

Лемма 4 доказана. 
Лемма 5. При условиях теоремь $1 \bar{b}_{n, k}=o_{k}(1)$.

Д о к а з а т е л ь с т в о. Пусть функция $\beta_{n}(k)$ такова, что при $k \rightarrow \infty, k \leqslant n$ справедливы соотношения $\beta_{n}(k) \rightarrow \infty$ и $\beta_{n}(k) \leqslant k^{1 / 4}$. В силу условия (4) и того, что $\left|a_{n}\right| \sqrt{n} / \sigma_{n}=d_{n} \rightarrow d, d \in[\varepsilon, A]$, функцию $\beta_{n}(k)$ можно выбрать так, чтобы $\beta_{n}(k) a_{n} \rightarrow 0$ при $k \rightarrow \infty, k \leqslant n$.

Тогда ввиду леммы 4

$$
\int_{-\beta_{n}(k)}^{0} x^{2} \mathrm{~d} \bar{F}_{n, k}(x)<\beta_{n}(k) \int_{-\beta_{n}(k)}^{0}|x| \mathrm{d} \bar{F}_{n, k}(x)<\beta_{n}(k)\left|\bar{a}_{n, k}\right|=o_{k}(1) .
$$

С другой стороны, согласно условию (5) и тому, что $\bar{F}_{n, k}(x)<F_{n}(x) F(x)$ при любых $x<0$ и $k \geqslant 1$,

$$
\begin{gathered}
\int_{-\infty}^{-\beta_{n}(k)} x^{2} \mathrm{~d} \bar{F}_{n, k}(x)=\beta_{n}^{2}(k) \bar{F}_{n, k}\left(-\beta_{n}(k)\right)-2 \int_{-\infty}^{-\beta_{n}(k)} x \bar{F}_{n, k}(x) \mathrm{d} x \\
\leqslant \beta_{n}^{2}(k) F_{n}(x) F\left(-\beta_{n}(k)\right)+2 \int_{-\infty}^{-\beta_{n}(k)}|x| F_{n}(x) F(x) \mathrm{d} x=o_{k}(1) .
\end{gathered}
$$

Следовательно, $\bar{b}_{n, k}=\int_{-\infty}^{0} x^{2} \mathrm{~d} \bar{F}_{n, k}(x)=o_{k}(1)$.

Д ок аза тель с т во те о ремы 1 . Покажем, что характеристическая функция

$$
f_{\bar{S}_{n, n}}\left(\frac{t}{\sigma_{n} \sqrt{n}}\right)=\mathbf{E} \exp \left\{\frac{i t \bar{S}_{n, n}}{\sigma_{n} \sqrt{n}}\right\}
$$

с ростом $n$ для всех $t$ из любого конечного интервала при $a_{n}>0$ стремится к характеристической функции закона $V_{1}(x ; d)$, а при $a_{n}<0-$ к характеристической функции закона $V_{2}(x ; d)$. Случаи $a_{n}>0$ и $a_{n}<0$ исследуем отдельно.

С л у ч а й $a_{n}>0, d \in\left[\varepsilon_{1}, A\right]$. Известно (см. [7, формула (52)]), что

$$
f_{\bar{S}_{n, n}}(t)=f_{n, 1}^{n}(t)+\sum_{k=1}^{n-1} f_{n, 1}^{k}(t) \bar{\varphi}_{n, n-k}(t)
$$

где

$$
f_{n, 1}(t)=\mathbf{E} e^{i t \xi_{n, 1}}, \quad \bar{\varphi}_{n, k}(t)=\int_{-\infty}^{0-}\left(1-e^{i t x}\right) \mathrm{d} \bar{F}_{n, k}(x) .
$$

Очевидно

$$
\sum_{k=1}^{n-1} f_{n, 1}^{k}\left(\frac{t}{\sigma_{n} \sqrt{n}}\right) \bar{\varphi}_{n, n-k}\left(\frac{t}{\sigma_{n} \sqrt{n}}\right)=\Sigma_{1}+\Sigma_{2},
$$

где

$$
\begin{aligned}
& \Sigma_{1}=\sum_{k=1}^{n-1} \exp \left\{\left(i t d_{n}-\frac{t^{2}}{2}\right) \frac{k}{n}\right\} \bar{\varphi}_{n, n-k}\left(\frac{t}{\sigma_{n} \sqrt{n}}\right), \quad d_{n}=\frac{\left|a_{n}\right| \sqrt{n}}{\sigma_{n}} \\
& \Sigma_{2}=\sum_{k=1}^{n-1}\left(f_{n, 1}^{k}\left(\frac{t}{\sigma_{n} \sqrt{n}}\right)-\exp \left\{\left(i t d_{n}-\frac{t^{2}}{2}\right) \frac{k}{n}\right\}\right) \bar{\varphi}_{n, n-k}\left(\frac{t}{\sigma_{n} \sqrt{n}}\right) .
\end{aligned}
$$


В силу леммы 4 при конечных $t$

$$
\left|\Sigma_{2}\right|<\frac{|t|^{3}}{n^{3 / 2}} \sum_{k=1}^{n-1} k(n-k)^{-1 / 2} o\left(\frac{1}{\sigma_{n} \sqrt{n}}\right)=|t|^{3} o_{n}(1)
$$

Далее,

$$
\begin{aligned}
\Sigma_{1}= & \frac{i t}{\sigma_{n} \sqrt{n}} \sum_{k=1}^{n-1} \exp \left\{\left(i t d_{n}-\frac{t^{2}}{2}\right) \frac{k}{n}\right\}\left(-\bar{a}_{n, n-k}\right) \\
& +\sum_{k=1}^{n-1} \exp \left\{\left(i t d_{n}-\frac{t^{2}}{2}\right) \frac{k}{n}\right\}\left(\bar{\varphi}_{n, n-k}\left(\frac{t}{\sigma_{n} \sqrt{n}}\right)-\left(-\frac{i t}{\sigma_{n} \sqrt{n}} \bar{a}_{n, n-k}\right)\right) .
\end{aligned}
$$

Отсюда, используя леммы 3 и 5 , находим

$$
\begin{aligned}
\Sigma_{1}= & \sum_{k=1}^{n-1} \exp \left\{\left(i t d_{n}-\frac{t^{2}}{2}\right) \frac{k}{n}\right\} \frac{i t}{\sigma \sqrt{n}} \\
& \times\left(\frac{\sigma}{\sqrt{2 \pi(n-k)}} e^{-d_{n}(1-k / n) / 2}-a_{n} \Phi\left(-d_{n} \sqrt{\frac{k}{n}}\right)\right)+\Sigma_{3}+\Sigma_{4},
\end{aligned}
$$

где

$$
\begin{aligned}
\left|\Sigma_{3}\right| \leqslant & \frac{|t|}{\sigma_{n} \sqrt{n}} \sum_{k=1}^{n-1} e^{-k t^{2} /(2 n)} \frac{\left|\varepsilon_{n, n-k}\right|}{\sqrt{n-k}} \leqslant \frac{|t|}{\sigma_{n} \sqrt{n}} \sum_{k=n-\sqrt{n+1}}^{n-1} \frac{1}{\sqrt{n-k}} \\
& +\frac{|t|}{\sigma_{n} \sqrt{n}}\left|\varepsilon_{n, \sqrt{n}}\right| \sum_{k=1}^{n-\sqrt{n}} \frac{1}{\sqrt{n-k}}=o_{n}(|t|), \\
\left|\Sigma_{4}\right| \leqslant & \left(\sum_{k=1}^{n-\sqrt{n}}+\sum_{k=n-\sqrt{n}+1}^{n-1}\right) \frac{t^{2}}{\sigma_{n}^{2} n} e^{-t^{2} k /(2 n)} \bar{b}_{n, n-k}=o_{n}\left(t^{2}\right) .
\end{aligned}
$$

В силу (38)-(42) имеем

$$
\begin{aligned}
& \sum_{k=1}^{n-1} f_{n, 1}^{k}\left(\frac{t}{\sigma_{n} \sqrt{n}}\right) \bar{\varphi}_{n, n-k}\left(\frac{t}{\sigma_{n} \sqrt{n}}\right) \\
& =\sum_{k=1}^{n-1} \exp \left\{\left(i t d_{n}-\frac{t^{2}}{2}\right) \frac{k}{n}\right\} \frac{i t}{\sigma_{n} \sqrt{n}} \\
& \quad \times\left(\frac{\sigma_{n}}{\sqrt{2 \pi(n-k)}} e^{-d_{n}(1-k / n) / 2}-a_{n} \Phi\left(-d_{n} \sqrt{\frac{k}{n}}\right)\right) \\
& +o_{n}\left(|t|+|t|^{3}\right) .
\end{aligned}
$$

Из (37) и (43) вытекает, что для всех $t$ из любого конечного интервала

$$
\lim _{n \rightarrow \infty} f_{\bar{S}_{n, n}}\left(\frac{t}{\sigma_{n} \sqrt{n}}\right)=e^{i t d-t^{2} / 2}+\lim _{n \rightarrow \infty} \sum_{k=1}^{n-1} i t \exp \left\{\left(i t d_{n}-\frac{t^{2}}{2}\right) \frac{k}{n}\right\}
$$




$$
\begin{aligned}
\times\left(\frac{1}{\sqrt{2 \pi(1-k / n)}} e^{-d^{2}(1-k / n) / 2}-d_{n} \Phi\left(-d_{n} \sqrt{\frac{k}{n}}\right) \frac{1}{n}\right) \\
=e^{i t d-t^{2} / 2}+i t \int_{0}^{1} \exp \left\{\left(i t d-\frac{t^{2}}{2}\right) u\right\} \\
\times\left(\frac{1}{\sqrt{2 \pi(1-u)}} e^{-d^{2}(1-u) / 2}-d \Phi(-d \sqrt{u})\right) \mathrm{d} u .
\end{aligned}
$$

Отсюда, так как

$$
\begin{aligned}
-i t d & \int_{0}^{1} e^{\left(i t d-t^{2} / 2\right) u} \Phi(-d \sqrt{1-u}) \mathrm{d} u \\
= & -i t d \int_{0}^{1} e^{\left(i t d-t^{2} / 2\right) u}\left(\frac{1}{2}-\frac{1}{\sqrt{2 \pi}} \int_{0}^{d \sqrt{1-u}} e^{-z^{2} / 2} \mathrm{~d} z\right) \mathrm{d} u \\
= & -\frac{d}{2 d+i t}\left(e^{i t d-t^{2} / 2}-1\right)-\frac{d^{2}}{\sqrt{2 \pi}(2 d+i t)} \int_{0}^{1} e^{-d^{2} u / 2} \frac{\mathrm{d} u}{\sqrt{u}} \\
& +\frac{d^{2} e^{-d^{2} / 2}}{\sqrt{2 \pi}(2 d+i t)} \int_{0}^{1} e^{(d+i t)^{2} u / 2} \frac{\mathrm{d} u}{\sqrt{1-u}}
\end{aligned}
$$

и

$$
-\frac{d^{2}}{\sqrt{2 \pi(2 d+i t)}} \int_{0}^{1} e^{-d^{2} u / 2} \frac{\mathrm{d} u}{\sqrt{u}}=-\frac{d}{2 d+i t} \sqrt{\frac{2}{\pi}} \int_{0}^{d} e^{-u^{2} / 2} \mathrm{~d} u
$$

получаем

$$
\begin{aligned}
& \lim _{n \rightarrow \infty} f_{\bar{S}_{n, n}}\left(\frac{t}{\sigma_{n} \sqrt{n}}\right) \\
& =\left(1-\frac{d}{i t+2 d}\right) e^{i t d-t^{2} / 2}+\frac{d}{i t+2 d}\left(1-\sqrt{\frac{2}{\pi}} \int_{0}^{d} e^{-u^{2} / 2} \mathrm{~d} u\right) \\
& \quad+\frac{1}{\sqrt{2 \pi}}\left(i t+\frac{d^{2}}{i t+2 d}\right) e^{-d^{2} / 2} \int_{0}^{1} e^{(d+i t)^{2} u / 2} \frac{\mathrm{d} u}{\sqrt{1-u}}
\end{aligned}
$$

Наконец, сравнивая (44) с (21), убеждаемся, что $f_{\bar{S}_{n, n}}\left(t /\left(\sigma_{n} \sqrt{n}\right)\right) \rightarrow$ $f_{V_{1}}(t), n \rightarrow \infty$, равномерно по $t$ из любого конечного интервала. Значит, соотношение (14) доказано.

С л у ч а й $a_{n}<0, d \in\left[\varepsilon_{1}, A\right]$. Поступая аналогично случаю $a_{n}>0, d \in\left[\varepsilon_{1}, A\right]$ и учитывая, что теперь $a_{n}<0$ и что, согласно лемме 3 ,

$$
\begin{gathered}
\bar{a}_{n, k}=-\frac{\sigma_{n}}{\sqrt{2 \pi k}} e^{-a_{n}^{2} k /\left(2 \sigma_{n}^{2}\right)}-\left|a_{n}\right|\left(\frac{1}{2}+\frac{1}{\sqrt{2 \pi}} \int_{0}^{\left|a_{n}\right| \sqrt{k} / \sigma_{n}} e^{-u^{2} / 2} \mathrm{~d} u\right)+\frac{\varepsilon_{n, k}}{\sqrt{k}} \\
=-\frac{\sigma_{n}}{\sqrt{n}}\left(\frac{1}{\sqrt{2 \pi k / n}} e^{-d_{n}^{2} k /(2 n)}\right. \\
\left.+d_{n}\left(\frac{1}{2}+\frac{1}{\sqrt{2 \pi}} \int_{0}^{\left|a_{n}\right| \sqrt{k} / \sigma_{n}} e^{-u^{2} / 2} \mathrm{~d} u\right)\right)+\frac{\varepsilon_{n, k}}{\sqrt{k}}
\end{gathered}
$$


получаем

$$
\begin{aligned}
\lim _{n \rightarrow \infty} & f_{\bar{S}_{n, n}}\left(\frac{t}{\sigma_{n} \sqrt{n}}\right)=e^{-i t d-t^{2} / 2}+\int_{0}^{1} \exp \left\{-\left(i t d+\frac{t^{2}}{2}\right) u\right\} \\
& \times\left[\frac{i t}{\sqrt{2 \pi(1-u)}} e^{-d^{2}(1-u) / 2}\right. \\
& \left.\quad+i t d\left(\frac{1}{2}+\frac{1}{\sqrt{2 \pi}} \int_{0}^{d \sqrt{1-u}} e^{-z^{2} / 2} \mathrm{~d} z\right)\right] \mathrm{d} u \\
= & e^{-i t d-t^{2} / 2}+\frac{i t}{\sqrt{2 \pi}} e^{-d^{2} / 2} \int_{0}^{1} e^{(i t-d)^{2} u / 2} \frac{\mathrm{d} u}{\sqrt{1-u}} \\
& -\frac{i t d}{2\left(i t d+t^{2} / 2\right)}\left(e^{-i t d-t^{2} / 2}-1\right)+\frac{i t d}{\left(i t d+t^{2} / 2\right)} \frac{1}{\sqrt{2 \pi}} \int_{0}^{d} e^{-z^{2} / 2} \mathrm{~d} z \\
& -\frac{i t d^{2}}{2 \sqrt{2 \pi}} \frac{1}{i t d+t^{2} / 2} \int_{0}^{1} e^{-\left(i t d+t^{2} / 2\right) u} e^{-d^{2}(1-u) / 2} \frac{\mathrm{d} u}{\sqrt{1-u}} \\
= & \left(1+\frac{d}{i t-2 d}\right) e^{-i t d-t^{2} / 2} \\
& +\frac{1}{\sqrt{2 \pi}}\left(i t+\frac{d^{2}}{i t-2 d}\right) e^{-d^{2} / 2} \int_{0}^{1} e^{(i t-d)^{2} u / 2} \frac{\mathrm{d} u}{\sqrt{1-u}} \\
& -\frac{d}{i t-2 d}\left(1+\sqrt{\frac{2}{\pi}} \int_{0}^{d} e^{-u^{2} / 2} \mathrm{~d} u\right) .
\end{aligned}
$$

Сравнение (46) с (26) показывает, что при $a_{n}<0, d \in\left[\varepsilon_{1}, A\right]$

$$
\lim _{n \rightarrow \infty} f_{\bar{S}_{n, n}}\left(\frac{t}{\sigma_{n} \sqrt{n}}\right)=f_{V_{2}}(t)
$$

равномерно по $t$ из любого конечного интервала. Это, в свою очередь, подтверждает справедливость соотношения (15). Теорема 1 доказана.

Для полноты изложения приведем доказательство соотношений (1), (3) и (9) методом характеристических функций. Конечно, будем считать, что выполнены условия (4) и (5).

Доказательст в о с оо тношения (9). Имеем $d_{n} \rightarrow \infty$, $a_{n}<0, n=1,2 \ldots$ Согласно (37),

$$
\begin{aligned}
& \int_{-\infty}^{\infty} e^{i t x} \mathrm{~d} \mathbf{P}\left\{\frac{\bar{S}_{n, n}}{\left|a_{n}\right|}<x\right\}=f_{\bar{S}_{n, n}}\left(\frac{t\left|a_{n}\right|}{\sigma_{n}^{2}}\right) \\
& =f_{n, 1}^{n}\left(\frac{t\left|a_{n}\right|}{\sigma_{n}^{2}}\right)+\sum_{k=1}^{n-1} f_{n, 1}^{k}\left(\frac{t\left|a_{n}\right|}{\sigma_{n}^{2}}\right) \bar{\varphi}_{n, n-k}\left(\frac{t\left|a_{n}\right|}{\sigma_{n}^{2}}\right) .
\end{aligned}
$$

Так как в окрестности точки $t=0$

$$
f_{n, 1}(t)=1-i t\left|a_{n}\right|-\frac{\sigma_{n}^{2} t^{2}}{2}+o\left(t^{2}\right),
$$


то при $|t| \leqslant \varepsilon$ и достаточно малом $\varepsilon$

$$
\left|f_{n, 1}(t)\right| \leqslant 1-\frac{\sigma_{n}^{2} t^{2}}{4}<e^{-\sigma_{n}^{2} t^{2} / 4}
$$

Следовательно, для $t \neq 0$ из любого конечного интеграла и достаточно большого $n$

$$
\left|f_{n, 1}^{n}\left(\frac{t\left|a_{n}\right|}{\sigma_{n}^{2}}\right)\right| \leqslant e^{-a_{n}^{2} n t^{2} /\left(4 \sigma_{n}^{2}\right)}=e^{-d_{n}^{2} t^{2} / 4}=o_{n}(1) .
$$

Нетрудно видеть, что

$$
\sum_{k=1}^{n-1} f_{n, 1}^{k}\left(\frac{t\left|a_{n}\right|}{\sigma_{n}^{2}}\right) \bar{\varphi}_{n, n-k}\left(\frac{t\left|a_{n}\right|}{\sigma_{n}^{2}}\right)=\sum_{k=1}^{n-n_{A}}+\sum_{k=n-n_{A}+1}^{n-1}=\Sigma_{1}+\Sigma_{2},
$$

где $n_{A}=\left[A \sigma_{n}^{2} / a_{n}^{2}\right], A$ - сколь угодно большое постоянное число и $[x]-$ целая часть числа $x$. Далее,

$$
\Sigma_{1}=\sum_{k=1}^{n-n_{A}} f_{n, 1}^{k}\left(\frac{t\left|a_{n}\right|}{\sigma_{n}^{2}}\right)\left(\frac{i t a_{n}^{2}}{\sigma_{n}^{2}}\right)+\Sigma_{1}^{\prime}+\Sigma_{1}^{\prime \prime}
$$

где

$$
\begin{aligned}
& \Sigma_{1}^{\prime}=\sum_{k=1}^{n-n_{A}} f_{n, 1}^{k}\left(\frac{t\left|a_{n}\right|}{\sigma_{n}^{2}}\right)\left[\bar{\varphi}_{n, n-k}\left(\frac{t\left|a_{n}\right|}{\sigma_{n}^{2}}\right)-\frac{i t a_{n}}{\sigma_{n}^{2}} \bar{a}_{n, n-k}\right], \\
& \Sigma_{1}^{\prime \prime}=\sum_{k=1}^{n-n_{A}} f_{n, 1}^{k}\left(\frac{t\left|a_{n}\right|}{\sigma_{n}^{2}}\right) \frac{i t a_{n}}{\sigma_{n}^{2}}\left[\bar{a}_{n, n-k}-a_{n}\right] .
\end{aligned}
$$

В силу (49) и леммы 5

$$
\left|\Sigma_{1}^{\prime}\right| \leqslant \frac{a_{n}^{2} t^{2}}{\sigma_{n}^{4}} \sum_{k=1}^{n-n_{A}} e^{-k a_{n}^{2} t^{2} /\left(4 \sigma_{n}^{2}\right)} \bar{b}_{n, n-k}=o_{n_{A}}\left(\frac{a_{n}^{2} t^{2}}{\sigma_{n}^{4}} \sum_{k=0}^{\infty} e^{-k a_{n}^{2} t^{2} /\left(4 \sigma_{n}^{2}\right)}\right)=o_{n}(1) .
$$

Далее, имея в виду (45) и равенство

$$
1-\Phi(x)=\frac{1}{x \sqrt{2 \pi}} e^{-x^{2} / 2}\left(1+O\left(\frac{1}{x^{2}}\right)\right), \quad x \rightarrow \infty,
$$

находим, что при $n_{A} \leqslant k \leqslant n-1$

$$
\begin{aligned}
\bar{a}_{n, k} & =-\frac{\sigma_{n}}{\sqrt{2 \pi k}} \exp \left\{-\frac{a_{n}^{2} k}{2 \sigma_{n}^{2}}\right\}-\left|a_{n}\right|\left(1-\frac{1}{\sqrt{2 \pi}} \int_{\left|a_{n}\right| \sqrt{k} / \sigma_{n}}^{\infty} e^{-u^{2} / 2} \mathrm{~d} u\right)+\frac{\varepsilon_{n, k}}{\sqrt{k}} \\
& =-\left|a_{n}\right|+r_{n, k}+\frac{\varepsilon_{n, k}}{\sqrt{k}}
\end{aligned}
$$

где

$$
\begin{aligned}
r_{n, k} & =\frac{\left|a_{n}\right|}{\sqrt{2 \pi}} \int_{\left|a_{n}\right| \sqrt{k} / \sigma_{n}}^{\infty} e^{-u^{2} / 2} \mathrm{~d} u-\frac{\sigma_{n}}{\sqrt{2 \pi}} \exp \left\{-\frac{a_{n}^{2} k}{2 \sigma_{n}^{2}}\right\} \\
& =O\left(\frac{\sigma_{n}^{3}}{a_{n}^{2} k^{3 / 2}} e^{-a_{n}^{2} k /\left(2 \sigma_{n}^{2}\right)}\right) .
\end{aligned}
$$


Тогда, учитывая (49), (55) и неравенство

$$
x^{\alpha} e^{-x} \leqslant e^{-\alpha} \alpha^{\alpha}, \quad x>0, \alpha>0,
$$

имеем

$$
\begin{aligned}
\left|\Sigma_{1}^{\prime \prime}\right| & \leqslant \frac{\left|a_{n}\right||t|}{\sigma_{n}^{2}} \sum_{k=1}^{n-n_{A}}\left(\left|r_{n, n-k}\right|+e^{-k a_{n}^{2} t^{2} /\left(4 \sigma_{n}^{2}\right)} \frac{\left|\varepsilon_{n, n-k}\right|}{\sqrt{n-k}}\right) \\
& =\frac{\left|a_{n}\right||t|}{\sigma_{n}^{2}} O\left(\sum_{k=n_{A}}^{n-1} \frac{\sigma_{n}^{3}}{a_{n}^{2} k^{3 / 2}} e^{-a_{n}^{2} k /\left(2 \sigma_{n}^{2}\right)}\right)+\frac{\left|\varepsilon_{n, n_{A}}\right|}{\sigma_{n}} \sum_{k=1}^{n-1} \frac{1}{\sqrt{k(n-k)}} \\
& =O\left(\frac{\left|a_{n}\right||t|}{\sigma_{n} n_{A}} \sum_{k=n_{A}}^{n-1} \frac{1}{\sqrt{k}} e^{-a_{n}^{2} k /\left(2 \sigma_{n}^{2}\right)}\right)+o_{n}(1) \\
& =O\left(\frac{\left|a_{n}\right||t|}{\sigma_{n} n_{A}}\left(1-e^{-a_{n}^{2} /\left(2 \sigma_{n}^{2}\right)}\right)^{-1 / 2}\right)+o_{n}(1) \\
& =O\left(\frac{|t|}{n_{A}}\right)+o_{n}(1)=o_{n}(1+A|t|) .
\end{aligned}
$$

Из (52), (53) и (57) вытекает, что

$$
\Sigma_{1}=\frac{i t a_{n}^{2}}{\sigma_{n}^{2}}\left(\frac{1-f_{n, 1}^{n-n_{A}+1}\left(\left|a_{n}\right| t / \sigma_{n}^{2}\right)}{1-f_{n, 1}\left(\left|a_{n}\right| t / \sigma_{n}^{2}\right)}-1\right)+o_{n}(1+A|t|) .
$$

$\mathrm{B}$ силу (48) при фиксированном $t, t \neq 0$, и $a_{n} \rightarrow 0$

$$
\frac{i t a_{n}^{2}}{\sigma_{n}^{2}}\left(1-f_{n, 1}\left(\frac{\left|a_{n}\right| t}{\sigma_{n}^{2}}\right)\right)^{-1}=\frac{2}{2-i t \sigma_{n}^{2}}\left(1+o_{n}(1)\right) .
$$

Следовательно,

$$
\Sigma_{1}=\frac{2}{2-i t \sigma_{n}^{2}}\left(1+o_{n}(1)\right)\left[1-f_{n, 1}^{n-n_{A}+1}\left(\frac{\left|a_{n}\right| t}{\sigma_{n}^{2}}\right)\right]+o_{n}(1+A|t|) .
$$

Отсюда, согласно (49), находим

$$
\Sigma_{1}=\frac{2}{2-i t \sigma_{n}^{2}}+O\left(\exp \left\{-\frac{a_{n}^{2} t^{2}}{4 \sigma_{n}^{2}}\left(n-n_{A}+1\right)\right\}\right)+o_{n}(1+A|t|) .
$$

Это означает, что при фиксированных $t, t \neq 0$, и $A$

$$
\lim _{n \rightarrow \infty} \Sigma_{1}=\frac{2}{2-i t \sigma^{2}} .
$$

Далее, учитывая (54)-(56), получаем

$$
\begin{aligned}
\left|\Sigma_{2}\right| & \leqslant \frac{\left|a_{n}\right||t|}{\sigma_{n}^{2}} \sum_{k=n-n_{A}+1}^{n-1} e^{-k a_{n}^{2} t^{2} /\left(4 \sigma_{n}^{2}\right)}\left(\left|a_{n}\right|+\left|r_{n, n-k}\right|+\frac{\left|\varepsilon_{n, n-k}\right|}{\sqrt{n-k}}\right) \\
& \leqslant \sum_{k=n-n_{A}}^{n-1} \frac{1}{\sigma_{n} \sqrt{k}}\left(\left|a_{n}\right|+\frac{\sigma_{n}^{3}}{a_{n}^{2}(n-k)^{3 / 2}}+\frac{\left|\varepsilon_{n, n-k}\right|}{\sqrt{n-k}}\right)=o_{n}(1) .
\end{aligned}
$$


Наконец, из соотношений (47), (50), (51), (58) и (59) следует, что

$$
\lim _{n \rightarrow \infty} \int_{-\infty}^{\infty} e^{i t x} \mathrm{~d} \mathbf{P}\left\{\frac{\bar{S}_{n, n}}{\left|a_{n}\right|}<x\right\}=\frac{2}{2-i t \sigma^{2}}
$$

Так как функция $2 /\left(2-i t \sigma^{2}\right)$ является характеристической функцией закона $1-e^{-2 x / \sigma^{2}}, x>0$, то (60) завершает доказательство соотношения (9).

Доказательст в о с о о тноше н ий (1) и (3). Пусть сначала $d_{n} \rightarrow \infty, a_{n}>0, n=1,2, \ldots$ Покажем, что тогда выполняется (3). Ввиду (37) имеем

$\mathbf{E} \exp \left\{i t \frac{\bar{S}_{n, n}-a_{n} n}{\sigma_{n} \sqrt{n}}\right\}=e^{-i t a_{n} n} f_{n, 1}^{n}\left(\frac{t}{\sigma_{n} \sqrt{n}}\right)+O\left(\frac{|t|}{\sigma_{n} \sqrt{n}} \sum_{k=1}^{n-1}\left|\bar{a}_{n, k}\right|\right)$.

Так как, согласно условиям (4) и (5), $e^{-i t a_{n} n} f_{n, 1}^{n}\left(t /\left(\sigma_{n} \sqrt{n}\right)\right) \longrightarrow e^{-t^{2} / 2}$, $n \rightarrow \infty$, то для доказательства соотношения (3) остается показать, что второе слагаемое правой части соотношения (61) стремится к нулю при $n \rightarrow \infty$.

Ввиду леммы 3 находим

$$
\frac{|t|}{\sigma_{n} \sqrt{n}} \sum_{k=1}^{n-1}\left|\bar{a}_{n, k}\right| \leqslant \frac{|t|}{\sigma_{n} \sqrt{n}} \sum_{k=1}^{n-1}\left(\frac{\sigma_{n}}{\sqrt{2 \pi k}} e^{-a_{n}^{2} k /\left(2 \sigma_{n}^{2}\right)}+a_{n} \Phi\left(-\frac{a \sqrt{k}}{\sigma_{k}}\right)+\frac{\left|\varepsilon_{n, k}\right|}{\sqrt{k}}\right) \text {. }
$$

Отсюда, учитывая неравенство $\Phi(-x)<(x \sqrt{2 \pi})^{-1} e^{-x^{2} / 2}, x>0$, получаем

$$
\frac{|t|}{\sigma_{n} \sqrt{n}} \sum_{k=1}^{n-1}\left|\bar{a}_{n, k}\right| \leqslant \frac{|t|}{\sigma_{n} \sqrt{n}} \sum_{k=1}^{n-1}\left(\frac{2 \sigma_{n}}{\sqrt{2 \pi k}} e^{-a_{n}^{2} k /\left(2 \sigma_{n}^{2}\right)}+\frac{\left|\varepsilon_{n, k}\right|}{\sqrt{k}}\right) .
$$

Воспользовавшись соотношением

$$
\frac{1}{\sqrt{\pi}} \sum_{k=1}^{\infty} \frac{z^{k}}{\sqrt{k}}=\frac{1}{\sqrt{1-z}}+\sum_{k=1}^{\infty} \rho_{k} z^{k}, \quad \rho_{k}=O\left(k^{-3 / 2}\right)
$$

находим, что при $d_{n} \rightarrow \infty$

$$
\frac{|t|}{\sqrt{2 \pi n}} \sum_{k=1}^{n-1} \frac{1}{\sqrt{k}} e^{-a_{n}^{2} k /\left(2 \sigma_{n}^{2}\right)}<\frac{|t|}{d_{n}}+O\left(\frac{|t|}{\sqrt{n}}\right)=o_{n}(|t|) .
$$

Далее, аналогично (59)

$$
\frac{|t|}{\sigma_{n} \sqrt{n}} \sum_{k=1}^{n-1} \frac{\left|\varepsilon_{n, k}\right|}{\sqrt{k}}=o_{n}(1)
$$

Из (62)-(64) вытекает, что при фиксированном $t$

$$
\frac{|t|}{\sigma_{n} \sqrt{n}} \sum_{k=1}^{n-1}\left|\bar{a}_{n, k}\right|=o_{n}(1) \text {. }
$$

Таким образом, соотношение (3) доказано. 
Пусть теперь $d_{n} \rightarrow \infty, n \rightarrow \infty$. Покажем, что выполняется соотношение (1). Доказательство соотношений

$$
\lim _{n \rightarrow \infty} f_{\bar{S}_{n, n}}\left(\frac{t}{\sigma_{n} \sqrt{n}}\right)=\int_{0}^{\infty} e^{i t x} \mathrm{~d} V_{i}(x ; 0),
$$

где $i=1$ при $a_{n}>0$ и $i=2$ при $a_{n}<0$, совпадает с доказательством соответствующих соотношений при $d \in\left[\varepsilon_{1}, A\right]$. Далее, так как при $d=0$

$$
V_{i}(x ; 0)=\frac{1}{\sqrt{2 \pi}} \int_{-\infty}^{x} e^{-u^{2} / 2} \mathrm{~d} u-\frac{1}{\sqrt{2 \pi}} \int_{-\infty}^{-x} e^{-u^{2} / 2} \mathrm{~d} u=\sqrt{\frac{2}{\pi}} \int_{0}^{x} e^{-u^{2} / 2} \mathrm{~d} u,
$$

$i=1,2$, то при $d_{n} \rightarrow 0$

$$
\lim _{n \rightarrow \infty} \mathbf{P}\left\{\frac{\bar{S}_{n, n}}{\sigma_{n} \sqrt{n}}<x\right\}=\sqrt{\frac{2}{\pi}} \int_{0}^{x} e^{-u^{2} / 2} \mathrm{~d} u,
$$

и соотношение (1) доказано.

\section{СПИСОК ЛИТЕРАТУРЫ}

1. Erdös P., Kac M. On certain limit theorems of the theory of probability. - Bull. Amer. Math. Soc., 1946, v. 52, p. 292-302.

2. Wald $A$. Limit distribution of the maximum and minimum of successive cumulative sums of random variables. - Bull. Amer. Math. Soc., 1947, v. 53, p. 142-153.

3. Chung K. L. Asymptotic distribution of the maximum cumulative sum of independent random variables. - Bull. Amer. Math. Soc., 1948, v. 54, p. 1162-1170.

4. Malmquist S. On certain confidence contours for distribution functions. - Ann. Math. Statist., 1954 , v. 25 , p. $523-533$.

5. Прохоров Ю.В. Переходные явления в процессах массового обслуживания. Литов. матем. сб., 1963, т. 3, № 1, с. 199-205.

6. Боровков A.A. Вероятностные процессы в теории массового обслуживания. М.: Наука, 1972, $368 \mathrm{c}$.

7. Нагаев C. В. Оценка скорости сходимости распределения максимума сумм независимых случайных величин. - Сиб. матем. журн., 1969, т. 10, № 3, с. 614-633.

8. Нагаев С. В. О скорости сходимости в одной граничной задаче. I. - Теория вероятн. и ее примен., 1970, т. 15 , в. 2, с. 179-199.

9. Алешкявичене $A . K$. Об аппроксимации распределения максимума сумм случайных величин с малым по абсолютной величине средним. - Литов. матем. сб., 1978 , т. 18 , № 2 , с. 5-20.

10. Градштейн И. С., Рыжик И. М. Таблицы интегралов, сумм, рядов и произведений. М. Физматгиз, 1962. 